INVITED REVIEW

100 YEARS OF INSULIN

\title{
Towards improved precision and a new classification of diabetes mellitus
}

\author{
Emma Ahlqvist1', Rashmi B Prasad1,2 and Leif Groop ${ }^{1,2}$ \\ 'Department of Clinical Sciences, Malmö, Lund University, Malmö, Sweden \\ IInstitute for Molecular Medicine Finland (FIMM), Helsinki University, Helsinki, Finland
}

Correspondence should be addressed to E Ahlqvist: emma.ahlqvist@med.lu.se

This paper forms part of a special section on 100 years of insulin. The guest editors for this section were James Cantley \& Decio Eizirik.

\begin{abstract}
Type 2 diabetes (T2D) is one of the fastest increasing diseases worldwide. Although it is defined by a single metabolite, glucose, it is increasingly recognized as a highly heterogeneous disease with varying clinical manifestations. Identification of different subtypes at an early stage of disease when complications might still be prevented could hopefully allow for more personalized medicine. An important step toward precision medicine would be to target the right resources to the right patients, thereby improving patient health and reducing health costs for the society. More well-defined disease populations also offer increased power in experimental, genetic and clinical studies. In a recent study, we used six clinical variables (glutamate decarboxylase autoantibodies, age at onset of diabetes, glycated hemoglobin, BMI and simple measures of insulin resistance and insulin secretion (so called HOMA estimates) to cluster adult-onset diabetes patients into five subgroups. These subgroups have been robustly reproduced in several populations worldwide and are associated with different risks of diabetic complications and responses to treatment. Importantly, the group with severe insulindeficient diabetes had increased risk of retinopathy and neuropathy, whereas the severe insulin-resistant diabetes group has the highest risk for diabetic kidney disease (DKD) and fatty liver. This emphasizes the key role of insulin resistance in the pathogenesis of DKD and fatty liver in T2D. In conclusion, this novel subclassification, breaking down T2D in clinically meaningful subgroups, provides the prerequisite framework for expanded personalized medicine in diabetes beyond what is already available for monogenic and to some extent type 1 diabetes.
\end{abstract}

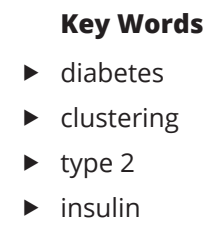

Journal of Endocrinology (2021) 252, R59-R70

\section{Heterogeneity of diabetes}

\section{Present official classification of diabetes}

Diabetes, particularly type 2 diabetes (T2D), is one of the fastest increasing diseases worldwide. However, under the term T2D, we lump together diseases with varying clinical manifestations, disease progression and risk of complications (Tuomi et al. 2014). Today, diabetes is diagnosed by measuring a single metabolite, glucose, which does not tell much about the nature of the disease. Hyperglycemia can develop if metabolism 
regulating glucose is disrupted, but this can be the consequence of multiple different metabolic processes which can vary between individuals (American Diabetes Association 2021). While some of these processes influence disease progression, drug response and development of complications, this knowledge covers only a small proportion of the clinical picture.

Diabetes is traditionally divided into type 1 diabetes (T1D) including latent autoimmune diabetes of the adult (LADA), T2D, specific types due to other causes (including monogenic diabetes syndromes, diseases of the exocrine pancreas and drug- or chemical-induced diabetes) and gestational diabetes.

Both T1D and LADA result from autoimmune destruction of beta cells, often, but not always, reflected by the presence of pancreatic autoantibodies in the blood (Regnell \& Lernmark 2017). Identification of such antibodies in plasma or serum is a strong indicator that the patient will eventually need insulin treatment to maintain glucose homeostasis (Buzzetti et al. 2017). Maturity Onset Diabetes of the Young includes monogenic form of diabetes accounting for about 3\% of diabetes diagnosed in individuals less than 30 years of age. An appropriate diagnosis requires sequencing of known diabetes genes (e.g. HNF1A, HNF4A and GCK), which is worthwhile as a correct diagnosis has major implications for choice of correct treatment (Pearson et al. 2006). Diabetes secondary to steroid use or medication, cystic fibrosis and hemochromatosis represent other diabetic subgroups.

After exclusion of these subgroups, about 10-15\% is considered to have T1D and $75-80 \%$ T2D. Since autoantibodies are not always measured and a genetic workup is not always possible, it is not uncommon that the T2D group includes patients with other misdiagnosed forms of diabetes. While the existence of two major diabetes types has been recognized for hundreds of years, the first description being attributed to the ancient Indian physician Sushruta and the surgeon Charaka (400-500 A.D.) (Frank 1957), the names and definitions have changed over time. In the early 20th century terms such as mild and severe diabetes or juvenile and maturity onset diabetes were used but these subgroups were considered to represent a spectrum of one and the same disease (Bennett 1985). In 1936, Harold Himsworth described the existence of insulin-sensitive and insensitive diabetes (Himsworth 1936, Karamanou et al. 2016). In 1950, RD Lawrence proposed a new clinical classification of diabetes patients into those who were probably not insulin deficient and those who definitely were (Bennett 1985). The terms T1D and T2D were coined in 1955 and have since been used for patient groups with different definitions relating to the main clinical characteristics of severity, age at onset, insulin sensitivity and insulin deficiency (Bennett 1985). In the 1970's, the discovery of autoantibodies to pancreatic islet cells led to the hallmarks of autoimmunity being added to describe T1D (Bottazzo et al. 1974). Still there is no definition that will allow all patients to be classified as either T1D or T2D (American Diabetes Association 2021). Some patients can show signs of both autoimmune destruction of beta cells and profound insulin resistance with features of the 'insulin resistance syndrome'. The large remaining group of T2D is still highly heterogeneous in terms of clinical characteristics, disease progression, treatment responses and risk of complications. Even before diabetes onset, elevated blood glucose, can be observed as impaired glucose tolerance and/or impaired fasting glucose. These risk factors show only a partial overlap, suggesting that they may result from different pathophysiological mechanisms (Unwin et al. 2002). It has become increasingly clear that T2D is a multifactorial disease resulting from the combined effects of genetic and environmental risk factors (Franks \& McCarthy 2016, Mahajan et al. 2018).

\section{Models of heterogeneity of type 2 diabetes}

Some models have been proposed to describe heterogeneity of T2D. In one of them, T2D is seen as a mixture of discrete subtypes that are caused by different mechanisms, where all individuals within each subtype have a homogeneous phenotype. Another model assumes that patients develop diabetes as a combination of many small defects in different pathways emphasizing the role of quantitative differences in metabolic pathways (McCarthy 2017, Pearson 2019).

The palette model can be considered a version of the second alternative. In the palette model, each pathway is imagined as a color and each patient given the hue of the combined pathways that are defective in the individual, which for most patients is assumed to be brown (McCarthy 2017). This model clearly has some benefits when applied to a common complex disease, but it also has drawbacks, especially when applied to research and clinical implementation as it does not recognize all measurable differences in clinical features between patients.

We have instead proposed an intermediate model which uses a broader brush and colors that reflect major clinical parameters instead of individual molecular pathways (Ahlqvist etal. 2020). The model still assumes that diabetes is caused by many overlapping mechanisms, but also assumes that individual patients have a predominant color. Therefore, dividing patients into shades of red, 
green and blue is more informative than considering them simply as shades of brown, although some patients present with intermediate colors. Different mechanisms will play dominating roles for different colors, for example some degree of insulin deficiency in leaner patients, whereas insulin resistance would dominate in more obese patients. In the following we will discuss this in more detail.

\section{A novel subclassification of adult-onset diabetes}

To dissect the heterogeneity of T2D, the All New Diabetics In Scania (ANDIS) study was initiated in 2008. The ANDIS project aims to include all newly diagnosed individuals with any type of diabetes in the Scania (Skåne) region in southern Sweden, within 1 year of diagnosis. To date, it includes $>20,000$ individuals representing $>90 \%$ of newly diagnosed patients. At registration, two measurements, that is of glutamate decarboxylase autoantibodies (GADA) and C-peptide, are added to standard measurements.

We stratified adult individuals into subtypes applying a data-driven approach using the most relevant, easily available, clinical variables for individuals with diabetes which include GADA, BMI, glycated hemoglobin $(\mathrm{HbA1c})$, age at diabetes onset, measures of beta cell function (HOMA2-B) and insulin resistance (HOMA2-IR) estimated from fasting glucose and C-peptide (Ahlqvist et al. 2018b) (Figure 1). These variables were selected based on the knowledge that diabetes develops when insulin secretion fails to meet the demands, for example due to insulin resistance (American Diabetes Association 2021). Hyperglycemia can thus result from either a deficient insulin secretion alone, as seen in T1D, or from more or less severe insulin resistance with insufficient insulin secretion as seen in many patients with T2D. This new classification is thus strongly based on measures describing the pathogenesis of diabetes, that is measures of insulin secretion and action.

We applied two different methods for clustering (Ahlqvist et al. 2018b). First, we used a method to determine the optimal number of clusters based on silhouette width followed by hierarchical clustering. In separate analyses in males and females, the optimal number of clusters was five, which was replicated in another cohort from the same region, the Scania Diabetes Registry (SDR). One cluster was completely defined by presence of GADA and was called severe autoimmune diabetes (SAID). Using k-means clustering, we could identify the four GADA-negative clusters of similar proportions with the same combination of clinical characteristics in four independent cohorts, including ANDIS and SDR.

\section{Differences between subgroups}

The five identified clusters showed different clinical characteristics, disease progression and outcomes. In addition to differences in clinical characteristics, the subgroups showed marked differences in the development of diabetic complications (Ahlqvist et al. 2018b). Based on these data, we proposed a new subclassification of T2D (Ahlqvist et al. 2018a,b, 2019), which is described and discussed below.

\section{Severe autoimmune diabetes}

The SAID cluster is defined by presence of GADA and thereby includes antibody positive individuals

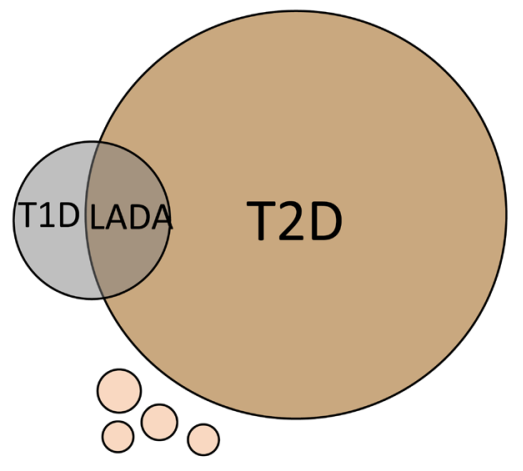

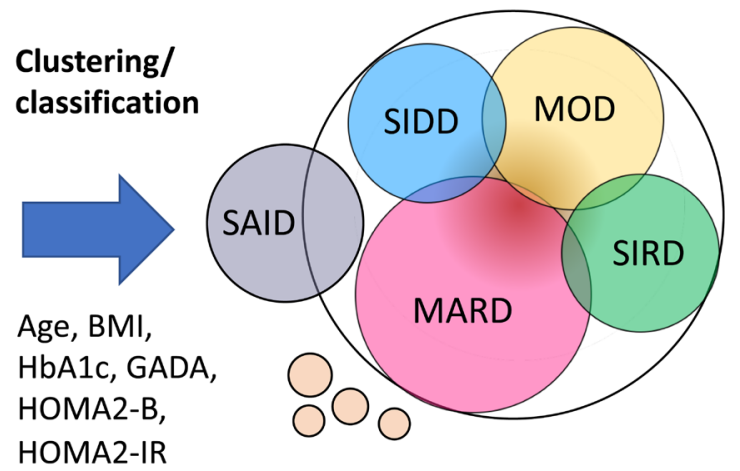

\section{Applications}

Clinic

Prediction of complications Choice of treatment

Research

Genetics

Mechanisms

Clinical trials

\section{Figure 1}

Subclassification of individuals with adult-onset diabetes. Individuals who would traditionally be classified as having T1D, LADA or T2D were subgrouped using a data-driven approach based on six commonly measured variables known to reflect central disease mechanisms. Individuals with known monogenic or secondary diabetes were excluded since they already have a well-defined diabetes type. The resulting subtypes could allow clinical support, with better prediction of risk of complications and response to therapy, and improved power and specificity in scientific endeavors by enabling studies in more homogeneous patient groups. 
traditionally referred to as T1D and LADA. This subgroup accounted for $6-8 \%$ of adult individuals in ANDIS. SAID was characterized by relatively early disease onset, poor insulin secretion, relatively low BMI and poor metabolic control (high HbA1c). While the group is defined by GADA positivity, any individual with autoantibodies associated with T1D should be considered autoimmune and assigned to this cluster. In a study performed in the German Diabetes Study (GDS), up to 7\% of GADA-negative patients were positive for islet cell antibodies (ICA) and/ or insulin autoantibodies, but these numbers might not be representative of the population, since autoimmune patients are oversampled in GDS (Zaharia et al. 2019). In a study performed in the Botnia region in Finland, only $0.5 \%$ of GADA-negative individuals with diabetes were positive for ICA and $0.6 \%$ for Islet antigen 2 antibodies (IA2) (Tuomi et al. 1999). In a subset of ANDIS, $1.3 \%$ of severe insulindeficient diabetes (SIDD) patients were positive for ZnT8A autoantibodies (Ahlqvist et al. 2018b) and in DIREVA, and $1.3 \%$ of SIDD patients were IA2 autoantibody positive (Mansour et al., MedRxiv, 2021).

\section{Severe insulin-deficient diabetes}

The SIDD cluster comprised $18-20 \%$ of studied individuals showing similar characteristics as the SAID group but without GADA. Like the SAID patients, they had poor insulin secretion (HOMA2-B), poor metabolic control (high HbA1c) and were moderately overweight.

Both the SAID and SIDD clusters had very high HbA1c at diagnosis, many of them presenting with ketoacidosis (31\% of SAID and $25 \%$ of SIDD). Both groups progressed more rapidly to insulin requirement and therapy than the other clusters. The SIDD group had the highest prevalence of early-onset diabetic retinopathy (Ahlqvist et al. 2018b). When scanned at an average of 135 days after diabetes diagnosis, 23\% already showed signs of mild retinopathy. Increased risk of retinopathy was also demonstrated in a Japanese cohort by Tanabe et al., where both SAID and SIDD had increased risk after adjustment for age and sex, but only the SAID group had higher risk after adjustment for modifiable risk factors including HbA1c (Tanabe et al. 2020).

These new diabetes subgroups were also explored in the GDS, where the low C-peptide secretory capacity of SIDD patients was confirmed using i.v. glucose tolerance tests (Zaharia et al. 2019). Intriguingly, the SIDD patients in GDS also showed higher prevalence of diabetic sensorimotor polyneuropathy and cardiac autonomic neuropathy already at diagnosis (12). Although metabolic control was markedly improved after 5 years, neuronal signaling and nerve function were not (Zaharia et al. 2019). These findings suggest that insulin deficiency could play a key role in the pathogenesis of retinopathy and neuropathy and raise the question whether SIDD patients would benefit from early, intense insulin therapy for the prevention of retinopathy and neuropathy. However, it remains to be shown whether increasing insulin or lowering of glucose would play the most important role.

\section{Severe insulin-resistant diabetes}

Patients in the third cluster, severe insulin-resistant diabetes (SIRD), accounted for about 15\% of all adult patients and were characterized by very high HOMA2-IR, HOMA2-B and high BMI but relatively good metabolic control as shown by a low HbA1c. They also had relatively late age at onset.

It is a common (mis)concept extrapolated from T1D that all microangiopathic complications, such as retinopathy, neuropathy and nephropathy, coincide also in T2D patients, which is clearly not the case. While retinopathy and neuropathy cluster together in SIDD, the SIRD subtype showed the highest risk of developing diabetic kidney disease (DKD) (Ahlqvist et al. 2018b).

TheSIRD subgroup had the lowest estimated glomerular filtration rate (eGFR) already at diabetes diagnosis and were thus already at increased risk of developing chronic kidney disease (CKD), macroalbuminuria and end-stage renal disease (ESRD). In the SDR cohort, SIRD patients had two times higher risk of developing CKD and macroalbuminura and almost five times higher risk of ESRD after adjustment for age and sex compared to MARD patients. These differences remained significant after exclusion of individuals with CKD at diagnosis and adjustment for initial eGFR and other commonly used variables (Ahlqvist et al. 2018b, 2020).

The increase in DKD in early stages of SIRD was also replicated in GDS (Zaharia et al. 2019). Further, SIRD was shown to be an independent risk factor for DKD in Japanese individuals with diabetes after adjustment for modifiable risk factors including eGFR, BMI and HbA1c (Tanabe et al. 2020). Dennis and colleagues analyzed the risk of developing CKD stage 3A during the first 4 years after diagnosis in two clinical trials, A Diabetes Outcome Progression Trial (ADOPT) and Rosiglitazone Evaluated for Cardiac Outcomesand Regulation of Glycaemia in Diabetes (RECORD) (15). They confirmed early signs of kidney disease in SIRD but could not replicate differences in progression after adjustment for initial eGFR possibly due to selection bias and follow-up time (Dennis et al. 2019). 
The relationship between insulin resistance and kidney disease is complex. Insulin resistance is a common and early alteration in CKD and almost universal in ESRD (Spoto et al. 2016). In healthy individuals, more than half of the plasma insulin is cleared by the kidney (Rabkin et al. 1984) and the increased HOMA2-IR in SIRD could therefore theoretically be the result of impaired C-peptide clearance due to reduced kidney function (Wallace et al. 2004). However, whole body estimates of insulin sensitivity (M) based on hyperinsulinaemic-euglycemic clamp is correlated well with HOMA2-IR based upon fasting glucose and C-peptide concentrations with the lowest M-values in SIRD patients both at diagnosis and after 5 years of follow-up (Zaharia et al. 2019).

SIRD patients also had the highest frequency of nonalcoholic fatty liver disease (NAFLD), as shown in ANDIS, defined by two pathological measurements of the liver enzyme, alanine aminotransferase and high BMI (Ahlqvist etal. 2018b).Zaharia etal. presented more accurate measures of liver affliction using MRI. In support of the findings in ANDIS, individuals from the SIRD cluster in GDS had the highest hepatocellular lipid content (HLC) (19\% compared to $<7 \%$ for other clusters) and the highest NAFLD fibrosis scores, fatty liver and aspartate aminotransferase-toplatelet ratio indices (Zaharia et al. 2019).

\section{Mild obesity-related diabetes}

The fourth cluster, mild obesity-related diabetes (MOD, 20-25\%) was also characterized by high BMI, but not by insulin resistance. Compared to the SIRD group, MOD patients had a much earlier onset and could be considered as a form of more 'healthy obesity' whereas the SIRD patients definitely represent 'unhealthy obesity' (Ahlqvist et al. 2018b).

\section{Mild age-related diabetes}

The fifth and largest cluster (39\%), mild age-related diabetes (MARD), had the latest onset of diabetes and the lowest BMI of the GADA-negative groups. The MARD group seems to have a relatively mild disease with few extreme characteristics and a low risk of diabetic complications (Ahlqvist et al. 2018b).

\section{Clinical implications and individualized treatment}

The recommendations for management of T2D issued by the American Diabetes Association and the European Association for the Study of Diabetes emphasize the

(c) 2021 Society for Endocrinology Published by Bioscientifica Ltd. Printed in Great Britain importance of personalized treatment based on patient characteristics and comorbidities (Davies et al. 2018). For patients with established cardiovascular disease or CKD, treatment with GLP-1RA or SGLT2i is recommended, but for other patients, selection between existing treatment options is focused on lowering glucose by stepwise addition of other medication than metformin, and guided by severity of hyperglycemia, obesity or cost restrictions.

The clinical differences between the subtypes suggest that choosing treatment that addresses the main underlying metabolic defect (e.g. insulin deficiency in SIDD and insulin resistance in SIRD) could also be beneficial.

Insulin resistance has long been recognized as a risk factor for $\mathrm{DKD}$ and shown to contribute to the development of disease through multiple mechanisms (Mitrofanova et al. 2019). Podocyte-specific deletion of the insulin receptor in mice causes albuminuria, together with histological features that recapitulate $\mathrm{DKD}$, even in a normoglycemic environment (Welsh et al. 2010). The fact that the SIRD subtype develops DKD in spite of relatively good metabolic control and low HbA1c levels indicates that treatment of these patients should primarily focus on enhancing insulin sensitivity and not simply lowering glucose. Individuals with SIRD show lower eGFR already at diagnosis suggesting that the pathological process starts before diabetes diagnosis and that such treatment should possibly be started before the onset of hyperglycemia.

The benefits of differential treatment in the subtypes remains to be tested. Some early evidence of such a benefit comes from the ADOPT and RECORD trials that explored three different treatments in GADA-negative patients (Dennis et al. 2019). The age-related (MARD) patients responded best to the insulin secretagogue sulfonylurea, whereas the insulin sensitizer rosiglitazone, a thiazolidinedione, showed the strongest effect in reducing HbA1c in the SIRD subtype (Dennis et al. 2019). In the ANDIS cohort, MARD and SIRD patients had been prescribed similar treatment in spite of considerable differences in risk of complications (Ahlqvist et al. 2018b). Unfortunately, the effect on eGFR was not tested in the ADOPT and RECORD trials, but thiazolidinediones have been shown to be effective in reducing albuminuria, even when achieving the same HbA1c targets (Schernthaner et al. 2004). Given the apparent key role of insulin resistance in the development of $\mathrm{DKD}$, this effect could be even stronger in SIRD patients.

These results are encouraging but we really need clinical trials that relate the treatment effects of other therapeutic agents to diabetic subgroups. The performance of insulin and GLP-1 agonists in the insulin-deficient subgroups and 
insulin sensitizers in SIRD needs to be tested, considering their potential for preventing complications like CKD and fatty liver. Among the insulin-deficient subgroups, SAID plays a particular role as it can be identified by measuring autoantibodies and thereby be targeted by insulin therapy early on.

\section{The novel sub-types in other populations}

A key question for us was whether the subclassification can be replicated and applicable in other populations. There are already a large number of studies published from different populations all over the world (Table 1). Most studies have been published from China and India but there are also several studies in other populations (Dennis et al. 2019, Zaharia et al. 2019, Zou et al. 2019, 2020, Anjana et al. 2020, Bello-Chavolla et al. 2020, Bennet et al. 2020, Kahkoska et al. 2020, Tanabe et al. 2020). While the results mostly indicate similar subtypes, albeit with slightly different distributions, it is clear that there are population differences that need to be taken into account when comparing clusters.

India and China are the most populated countries with the fastest growing economies in the world and the prevalence of diabetes has tripled in China and doubled in India in less than two decades (IDF 2019). Both Indians and Chinese develop diabetes at younger age and at lower BMI compared to Caucasians (Shelgikar et al. 1991, Boffetta et al. 2011, IDF 2019). This does not necessarily indicate that subtypes with earlier onset and lower BMI should be more common, but rather that the cluster variables are on a different scale. For example, the body composition of South Asians in general is different compared to the Caucasian population. For a given BMI, the fat percentage is significantly higher in South Asians than Caucasians and associated with more severe insulin resistance (Yajnik \& Yudkin 2004, Boffetta et al. 2011).

In Chinese populations, the novel diabetes clustering was robustly replicated in 2316 newly diagnosed diabetic participants of the National Diabetes and Metabolic Disorders Study (CNDMDS) and 685 participants from the National Health and Nutrition Examination Survey (NHANES III) from China and US (Zou et al. 2019). In the absence of GADA, four k-means clusters could be identified using age at diagnosis, BMI, HbA1c (or alternatively mean plasma glucose), HOMA2-B and HOMA2-IR. MARD comprised nearly half the participants in both studies, followed by MOD, whereas SIRD and SIDD were least prevalent. Notably, SIDD was more prevalent in Chinese than in Caucasian populations, a finding also replicated in other Asian studies. The clusters have also been replicated in a large Chinese cross-sectional study with 15,772 patients with adult-onset diabetes that again identified the above five subgroups (Li et al. 2020). Another study based on 5414 patients from the National Clinical Research Center for Metabolic Diseases Diabetes Center in China found only three out of the five ANDIS clusters (SAID, SIDD and MARD) based on the same clustering parameters. However, seven subgroups were obtained which included all the five ANDIS subgroups and two new subtypes (UARD and IRD) when other clustering variables such as triglycerides and uric acid were added to the cluster analysis (Xiong et al. 2021). As previously mentioned, similar findings were reported from Japan, replicating the increased risk of retinopathy in SIDD and DKD in SIRD (Tanabe et al. 2020).

Given that India is often referred to as the 'World Capital for Diabetes', the topic has also been addressed in India (Prasad et al. 2019). We recently confirmed the generalizability of the classification in an Indian population diagnosed at less than 45 years of age (Prasad et al. 2021), where we applied the clustering method to 1612 T2D patients $\sim 10$ years post diagnosis. The insulindeficient SIDD subgroup was more common in India whereas the mild obese MOD was more common in Europe. The four clusters recapitulated the clinical characteristics observed in the Swedish and other European populations, suggesting generalizability of the classification in nonEuropean populations. This was also replicated in three other studies from various parts of India (Prasad et al. 2021).

From 50 diabetes centers across India, 19,085 individuals with $\mathrm{T} 2 \mathrm{D}$ and duration less than 5 years, were selected in the INdia-Scotland Partnership for pRecision mEdicine inDiabetes (INSPIRED) study. However, here the authors, to some extent, applied different variables for clustering with clear effects on results. When including lipids (triglycerides and HDL-cholesterol) in the panel of clustering variables, the authors identified two similar (SIDD and MARD) and two novel subgroups called combined insulin-resistant and -deficient diabetes and insulin-resistant obese diabetes (Anjana et al. 2020).

Another study comprising 1293 participants from South Asians Living in America (MASALA) and a MultiEthnic Study of Artherosclerosis cohorts also identified five subgroups which were characterized by older age at onset (43\%), hyperglycemia (26\%), severe obesity (20\%), younger age at onset (1\%) and requiring insulin medication use (9\%). The severe hyperglycemia group was more common in South Asians whereas older age at onset group was more common in all the other ethnicities (Bancks et al. 2021). 


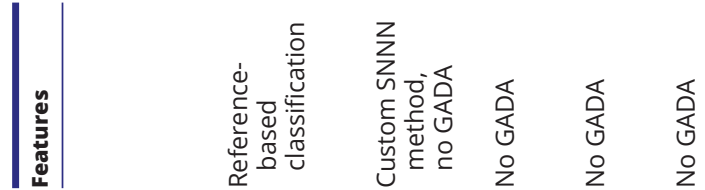

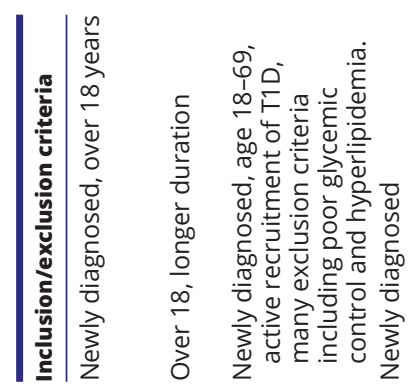

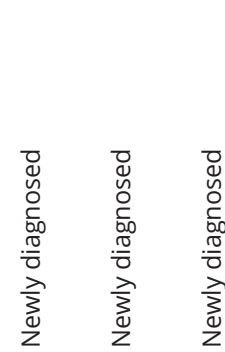

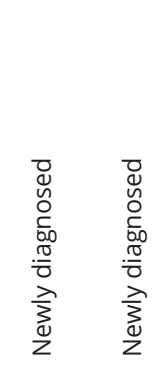

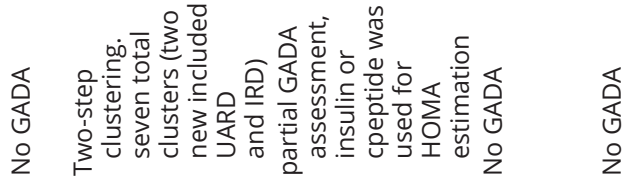
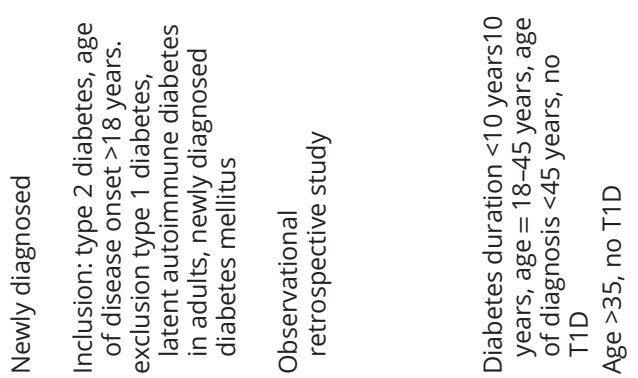

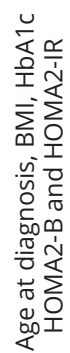

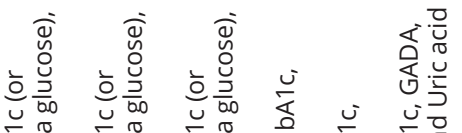

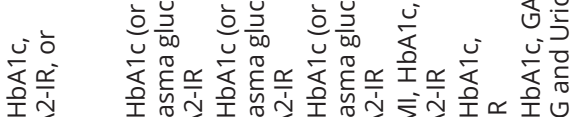

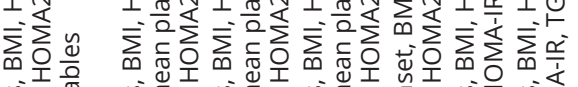

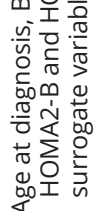

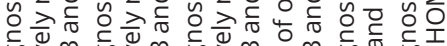

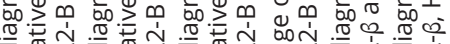

$\begin{array}{llll} & \\ 0 & \end{array}$

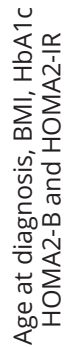
vํ.

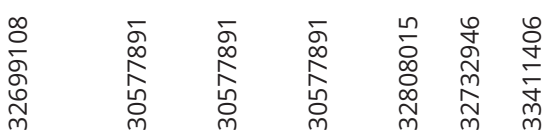

尊

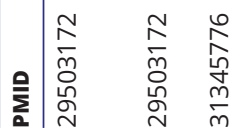

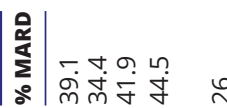

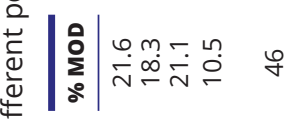

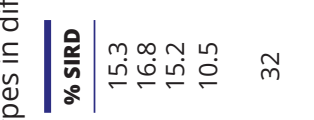

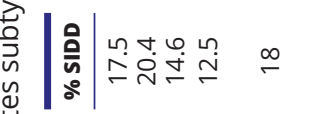

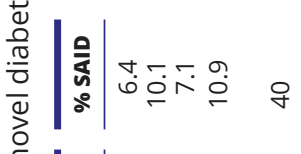

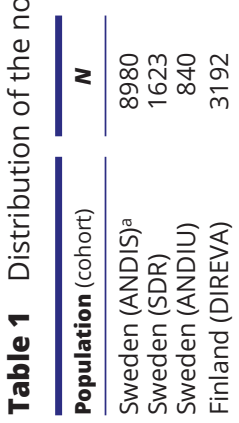

\section{$\stackrel{\infty}{\ln } \underset{\ln }{\infty} \stackrel{\infty}{\infty}$}

$\infty$

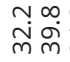

ธேำ

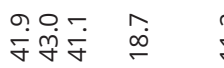

$1 \quad 1 \quad 1 \quad$ กู

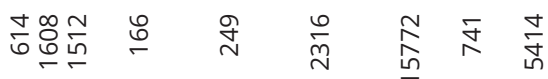

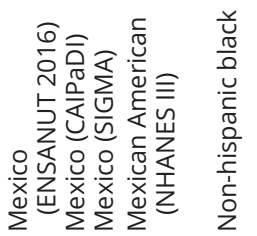

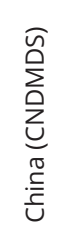

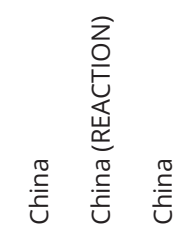

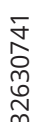

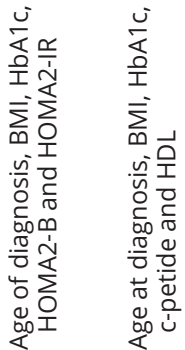

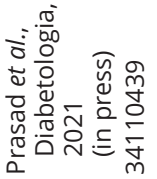

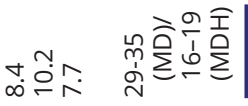

…ำ.

mิm min

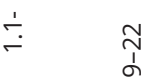

숭ํㅇ

$\operatorname{nin} \theta$

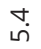

ํㅗำ

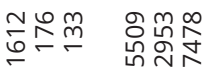

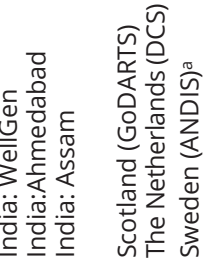


In the Mexican population, the same clusters as in Ahlqvist et al., were identified in several different cohorts. The clinical variables followed the expected pattern for each subgroup in all cohorts but the prevalence of each subtype was dramatically different compared to European populations with most individuals belonging to the SIDD and MOD subtypes (Bello-Chavolla et al. 2020).

\section{Clustering efforts using alternative approaches}

The novel classification is based on measures of GADA and c-peptide that are not available for many existing research cohorts and clinical studies. This has led to replication efforts and follow-up studies that use surrogate variables for classification. The validity of such studies depends on the variables used and the possibility to verify that the resulting clusters really correspond to the groups identified in Ahlqvist et al. HOMA2 can be calculated using either fasting c-peptide or insulin. Using fasting insulin should be adequate in treatment naïve patients but would be problematic in individuals treated with insulin. The HOMA2 measures seem to be essential for the classification, especially for distinguishing the SIRD subgroup. Kahkoska et al. tried to validate the novel subtypes in the DEVOTE, LEADER and SUSTAIN-6 cardiovascular outcomes trials using ANDIS as reference to assign study participants into clusters based on age at diabetes diagnosis, HbA1c and BMI only (Kahkoska et al. 2020). They found the highest risk for cardiovascular events in the cluster of participants with high HbA1c and low BMI that most closely resembled SIDD but could not replicate the increased risk of kidney disease in the cluster that most resembled SIRD. To test how well these subgroups would correspond to our subtypes, we used the same method of classification in ANDIS, where individuals in the SIDD-like group included 90\% SIDD patients but the SIRD-like group only $40 \%$ SIRD patients, probably explaining the lack of replication of the kidney phenotype (Ahlqvist et al. 2020).

Bello-Chavola et al. used self-normalizing neural networks (SNNN) for classification using surrogate variables (Bello-Chavolla et al. 2020). This method improved classification when c-peptide-based HOMA and HbA1c were missing, but still had inferior performance for the SIRD subtype. Importantly, any alternative methods should be properly verified before conclusions are drawn from the resulting clusters.

Another potential problem is the dramatic change in HbA1c levels observed in the SIDD group after treatment
(Ahlqvist et al. 2018b). While the average HbA1c levels remain higher in the insulin-deficient subtypes years after diagnosis, using $\mathrm{HbA} 1 \mathrm{c}$ from treated patients could make it more difficult to correctly identify the SIDD subtype.

Related questions are whether the clustering method gives the same results at diagnosis and after a longer duration of diabetes, to what extent individuals will move from one cluster to another and how this would affect their risk profile. In the DIREVA study from Finland, we clustered both individuals registered within 2 years after diagnosis and individuals with longer duration of diabetes with similar results. While this supports the robustness of clusters and indicates limited influence of disease duration, this needs to be studied in cohorts with repeated measurements of the clustering variables. One of the few cohorts that has had the opportunity to do this is GDS, where $23 \%$ changed cluster allocation 5 years after diagnosis (Zaharia et al. 2019). However, this analysis is based on only 367 individuals that were not representative of all diabetes patients since the cohort is selectively recruiting patients with several inclusion and exclusion criteria affecting the cluster distribution, including exclusion of individuals with poor metabolic control and hyperlipidemia (Szendroedi et al. 2016). Since the cluster variables can change over time (except age at diabetes diagnosis) it is obvious that some individuals could also change cluster. The effect of such a change depends on the reason of which could be, for example, because they were on the border between subtypes to start with or because treatment and lifestyle affected the cluster variables.

Some studies have also tried to build on the clustering by adding additional variables. This could be a valuable possibility to improve precision of the classification depending on the relevance of the added parameters. Clustering with additional variables could also allow further subgrouping but the value of such groups depends on the clinical relevance of the parameters and resulting groups. The Innovative Medicines Initiative (IMI)Rhapsody consortium replicated the ANDIS subtypes in three European cohorts (including ANDIS) using only c-peptide instead of HOMA and adding HDL cholesterol to the cluster variables. This approach led to a split of the MARD group into two groups that they called mild diabetes (MD) and MD with high HDL (MDH), of which MDH had the slowest glycemic deterioration of all clusters (Slieker et al. 2021).

The progression to diabetes is a slow process and is preceded by a phase of prediabetes which is characterized by intermediate hyperglycemia and is often undiagnosed. Some diabetes complications such as DKD in SIRD could 
immensely benefit from early interventions and indeed preventive action. Prediabetes phenotyping could be immensely beneficial in this regard. An elegant study by Wagner et al. has implemented such an approach in 899 participants from TUEF/TULIP study and replicated in Whitehall II cohort $(n=6810)$ using similar variables, that is glycemia during glucose challenge, insulin sensitivity, insulin secretion, fasting insulin, fasting triglycerides, waist circumference, hip circumference, BMI and HDLcholesterol. Wagner et al. reported six clusters with different propensities to develop diabetes, most of which could be suggestively mapped to the ANDIS subgroups. This would be invaluable in early identification of individuals with high future risk for complications and therefore prevention (Wagner et al. 2021).

\section{Genetics of novel subgroups and implications for etiology}

While the phenotype clearly differs between clusters, the question arises whether they also differ etiologically. Probably the best way to answer this question is to examine whether they also differ genetically and whether such genetic differences can be linked to mechanistic etiologic differences.

More than 400 loci affecting risk of T2D have been identified; many of them are also associated with clinical and metabolic subphenotypes such as BMI, insulin secretion and insulin resistance (Mahajan et al. 2018). These genetic associations may help to shed light on what pathways are involved in disease etiology. To address this, we have recently performed a Genome Wide Association Study (GWAS) in 7010 patients with adult-onset diabetes and 2744 age-matched healthy controls (Mansour et al., MedRxiv, 2020).

The results confirmed previous findings that SIRD is not associated with the well-established T2D locus in TCF7L2 (Grant et al. 2006, Saxena et al. 2006) nor with GRS for insulin secretion (Ahlqvist et al. 2018b). A genetic variant in PNPLA3 has also been shown to be associated with HLC and the SIRD subtype. In SIRD, the G-allele carriers had higher circulating free fatty acid concentrations and greater adipose tissue insulin resistance compared with noncarriers (Zaharia et al. 2020). These results clearly show that SIRD is a distinct subtype with at least partially different etiology.

Genetic variants have also been used as clustering variables. These studies have used multi-variant-trait association patterns from published GWAS of various diabetes related traits to group loci with shared disease mechanisms. This strategy is based on the assumption that genetic loci that act along a shared pathway will have similar impact on subphenotypes (Mahajan et al. 2018, Udler et al. 2018). Udler et al. used a soft clustering method to group variant-trait associations from publicly available GWAS for 94 known T2D variants and 47 T2D-related traits (Udler et al. 2018). Five SNP clusters were obtained, two of which were related to insulin deficiency and three to insulin resistance. These were then used to assign patients into groups with differing genetic risk profiles. When comparing the SNPs included in the pathway clusters with associations in ANDIS, variants associated with SIDD had the strongest weights in the proinsulin/beta cell genetic clusters, the genetic obesity cluster shared associations with MOD, whereas the genetically obtained insulin resistance cluster shared genetic associations with SIRD (Udler et al. 2018). While this approach to subclassification of patients has the advantage of being robust over time, focusing solely on genetic risk has several disadvantages since it does not reflect the environmental risk factors. Heritability determines less than half of the risk of T2D and the identified risk loci for T2D only explain a small proportion of the heritability. Thus, at present these methods do not seem to have the same clinical potential as the ANDIS-based subtypes.

\section{Interpretations and conclusions}

Easily measurable clinical parameters can be used to subclassify individuals with T2D into five groups with different clinical characteristics and disease progression. These clusters have been shown to be relatively stable over time and reproducible in several populations. Classification can be done also for single individuals and selected cohorts using a reference population, greatly expanding the utility of the method and opening avenues for personalized and stratified therapy.

However, the mixed results of studies trying to replicate the clustering using alternative methods emphasizes the importance of predefined inclusion criteria and clustering variables. Especially, HOMA measurements seem to be essential for proper cluster assignment and identification of the high-risk SIRD group and so far no alternative variables have been able to substitute for c-peptide or insulin measurements. Using a reference for classification can solve some of the problems associated with smaller selected cohorts but requires large-high-quality, -population-specific cohorts as reference. For European populations, ANDIS 
can be used as such a reference using, for example, nearest centroid-based methods of classification. Tools for referencebased classification could be expanded to include reference populations of different ethnicity, as well as adaptations for different treatment status and duration of diabetes.

It is clear that SAID, SIDD and SIRD would benefit for choice of treatment from a cluster-based definition of the subgroups. Insulin and insulin secretagogues are likely the therapy of choice for SAID and SIDD, and insulin sensitizers for SIRD, but this should still be demonstrated in large intervention trials. Metformin seems to be a good choice for MOD and preliminary data have suggested that sulfonylureas performs well in MARD. However, this needs to be tested in a clinical setting taking the risk of hypoglycemia associated with sulfonylureas in elderly patients into account. Using continuous variables could provide even better prediction of specific complications and response to treatment (Dennis et al. 2019) and might be a valuable complement to stratification. The additional cost of measuring c-peptide and GADA may hamper the use of this classification in poorer areas of the globe. However, because c-peptide is an independent predictor of, for example, kidney disease (Ahlqvist et al. 2020), this cost could be considered small in comparison to the cost of treating complications.

The clustering also opens up new avenues for research allowing better definitions of underlying pathogenic defects. The current classification should not be considered as final despite extensive replication. In the future, it could be possible to refine the ANDIS stratification further by inclusion of additional cluster variables, such as biomarkers, genotypes or genetic risk scores. More advanced methods using artificial intelligence also offer a promising avenue toward subtypes with even higher resolution and better predictive power.

This novel subclassification nevertheless potentially represents a valuable clinical tool to aid clinical decisions.

\section{Declaration of interest}

$\mathrm{L} G$ is the guarantor. The other authors have no conflicts of interest.

\section{Funding}

This work was supported by grants from the Swedish Research Council (2017-02688), Diabetes Wellness Sweden (25-420 PG) and the Swedish Heart-Lung Foundation.

\section{Author contribution statement}

E A, R P and L G wrote the manuscript.

(C) 2021 Society for Endocrinology Published by Bioscientifica Ltd. Printed in Great Britain

\section{References}

Ahlqvist E, Prasad RB, Tuomi T, Rosengren A \& Groop L 2018a Novel diabetes subgroups - authors' reply. Lancet: Diabetes and Endocrinology 6 440-441. (https://doi.org/10.1016/S2213-8587(18)30139-6)

Ahlqvist E, Storm P, Karajamaki A, Martinell M, Dorkhan M, Carlsson A, Vikman P, Prasad RB, Aly DM, Almgren P, et al. 2018b Novel subgroups of adult-onset diabetes and their association with outcomes: a datadriven cluster analysis of six variables. Lancet: Diabetes and Endocrinology 6 361-369. (https://doi.org/10.1016/S2213-8587(18)30051-2)

Ahlqvist E, Tuomi T \& Groop L 2019 Clusters provide a better holistic view of type 2 diabetes than simple clinical features. Lancet: Diabetes and Endocrinology 7 668-669. (https://doi.org/10.1016/S22138587(19)30257-8)

Ahlqvist E, Prasad RB \& Groop L 2020 Subtypes of type 2 diabetes determined from clinical parameters. Diabetes 69 2086-2093. (https:// doi.org/10.2337/dbi20-0001)

American Diabetes Association 2021 2. Classification and diagnosis of diabetes: standards of medical care in diabetes-2021. Diabetes Care $\mathbf{4 4}$ S15-S33. (https://doi.org/10.2337/dc21-S002)

Anjana RM, Baskar V, Nair ATN, Jebarani S, Siddiqui MK, Pradeepa R, Unnikrishnan R, Palmer C, Pearson E \& Mohan V 2020 Novel subgroups of type 2 diabetes and their association with microvascular outcomes in an Asian Indian population: a data-driven cluster analysis: the INSPIRED study. BMJ Open Diabetes Research and Care $\mathbf{8} 1506$. (https://doi.org/10.1136/bmjdrc-2020-001506)

Bancks MP, Bertoni AG, Carnethon M, Chen H, Cotch MF, Gujral UP, Herrington D, Kanaya AM, Szklo M, Vaidya D, et al. 2021 Association of diabetes subgroups with race/ethnicity, risk factor burden and complications: the MASALA and MESA studies. Journal of Clinical Endocrinology and Metabolism 106 e2106-e2115. (https://doi. org/10.1210/clinem/dgaa962)

Bello-Chavolla OY, Bahena-López JP, Vargas-Vázquez A, Antonio-Villa NE, Márquez-Salinas A, Fermín-Martínez CA, Rojas R, Mehta R, CruzBautista I, Hernández-Jiménez S, et al. 2020 Clinical characterization of data-driven diabetes subgroups in Mexicans using a reproducible machine learning approach. BMJ Open Diabetes Research and Care $\mathbf{8}$ e001550. (https://doi.org/10.1136/bmjdrc-2020-001550)

Bennet L, Nilsson C, Mansour-Aly D, Christensson A, Groop L \& Ahlqvist E 2020 Adult-onset diabetes in Middle Eastern immigrants to Sweden: novel subgroups and diabetic complications-the all new diabetes in Scania cohort diabetic complications and ethnicity. Diabetes/Metabolism Research and Reviews 37 e3419. (https://doi.org/10.1002/dmrr.3419)

Bennett PH 1985 Basis of the present classification of diabetes. Advances in Experimental Medicine and Biology 189 17-29. (https://doi. org/10.1007/978-1-4757-1850-8_2)

Boffetta P, McLerran D, Chen Y, Inoue M, Sinha R, He J, Gupta PC, Tsugane S, Irie F, Tamakoshi A, et al. 2011 Body mass index and diabetes in Asia: a cross-sectional pooled analysis of 900,000 individuals in the Asia cohort consortium. PLoS ONE 6 e19930. (https://doi.org/10.1371/ journal.pone.0019930)

Bottazzo GF, Florin-Christensen A \& Doniach D 1974 Islet-cell antibodies in diabetes mellitus WITH autoimmune polyendocrine deficiencies. Lancet 2 1279-1283. (https://doi.org/10.1016/s0140-6736(74)90140-8)

Buzzetti R, Zampetti S \& Maddaloni E 2017 Adult-onset autoimmune diabetes: current knowledge and implications for management. Nature Reviews: Endocrinology 13 674-686. (https://doi.org/10.1038/ nrendo.2017.99)

Davies MJ, D’Alessio DA, Fradkin J, Kernan WN, Mathieu C, Mingrone G, Rossing P, Tsapas A, Wexler DJ \& Buse JB 2018 Management of hyperglycemia in type 2 diabetes, 2018. A consensus report by the American Diabetes Association (ADA) and the European Association for the Study of Diabetes (EASD) Diabetes Care 41 2669-2701. (https:// doi.org/10.2337/dci18-0033)

Dennis JM, Shields BM, Henley WE, Jones AG \& Hattersley AT 2019 Disease progression and treatment response in data-driven subgroups 
of type 2 diabetes compared with models based on simple clinical features: an analysis using clinical trial data. Lancet: Diabetes and Endocrinology 7 442-451. (https://doi.org/10.1016/S22138587(19)30087-7)

Frank LL 1957 Diabetes mellitus in the texts of old Hindu medicine (Charaka, Susruta, Vagbhata). American Journal of Gastroenterology 27 76-95.

Franks PW \& McCarthy MI 2016 Exposing the exposures responsible for type 2 diabetes and obesity. Science 354 69-73. (https://doi.org/10.1126/ science.aaf5094)

Grant SF, Thorleifsson G, Reynisdottir I, Benediktsson R, Manolescu A, Sainz J, Helgason A, Stefansson H, Emilsson V, Helgadottir A, et al. 2006 Variant of transcription factor 7-like 2 (TCF7L2) gene confers risk of type 2 diabetes. Nature Genetics 38 320-323. (https://doi.org/10.1038/ ng1732)

Himsworth HP 1936 Diabetes mellitus: its differentiation INTO insulinsensitive and insulin-insensitive types. Lancet 28 127-130.(https://doi. org/10.1136/bmj.2.3942.188 )

IDF 2019 IDF Diabetes Atlas, 9th ed. International Diabetes Federation.

Kahkoska AR, Geybels MS, Klein KR, Kreiner FF, Marx N, Nauck MA, Pratley RE, Wolthers BO \& Buse JB 2020 Validation of distinct type 2 diabetes clusters and their association with diabetes complications in the DEVOTE, LEADER and SUSTAIN-6 cardiovascular outcomes trials. Diabetes, Obesity and Metabolism 22 1537-1547. (https://doi.org/10.1111/ dom.14063)

Karamanou M, Protogerou A, Tsoucalas G, Androutsos G \& PoulakouRebelakou E 2016 Milestones in the history of diabetes mellitus: the main contributors. World Journal of Diabetes 7 1-7. (https://doi. org/10.4239/wjd.v7.i1.1)

Li X, Yang S, Cao C, Yan X, Zheng L, Zheng L, Da J, Tang X, Ji L, Yang X, et al. 2020 Validation of the Swedish diabetes re-grouping scheme in adult-onset diabetes in China. Journal of Clinical Endocrinology and Metabolism 105 dgaa524. (https://doi.org/10.1210/clinem/dgaa524)

Mahajan A, Wessel J, Willems SM, Zhao W, Robertson NR, Chu AY, Gan W, Kitajima H, Taliun D, Rayner NW, et al. 2018 Refining the accuracy of validated target identification through coding variant fine-mapping in type 2 diabetes. Nature Genetics 50 559-571. (https://doi.org/10.1038/ s41588-018-0084-1)

Mansour Aly D, Dwivedi OP, Prasad RB, Käräjämäki A, Hjort R, Thangam M, Åkerlund M, Mahajan A, Udler MS, Florez JC, et al. 2021 Genome-wide association analyses highlight etiological differences underlying newly defined subtypes of diabetes. Nature Genetics 53 1534-1542. (https:// doi.org/10.1038/s41588-021-00948-2)

McCarthy MI 2017 Painting a new picture of personalised medicine for diabetes. Diabetologia 60 793-799. (https://doi.org/10.1007/s00125-0174210-x)

Mitrofanova A, Sosa MA \& Fornoni A 2019 Lipid mediators of insulin signaling in diabetic kidney disease. American Journal of Physiology: Renal Physiology 317 F1241-F1252. (https://doi.org/10.1152/ ajprenal.00379.2019)

Pearson ER 2019 Type 2 diabetes: a multifaceted disease. Diabetologia 62 1107-1112. (https://doi.org/10.1007/s00125-019-4909-y)

Pearson ER, Flechtner I, Njolstad PR, Malecki MT, Flanagan SE, Larkin B, Ashcroft FM, Klimes I, Codner E, Iotova V, et al. 2006 Switching from insulin to oral sulfonylureas in patients with diabetes due to Kir6.2 mutations. New England Journal of Medicine 355 467-477. (https://doi. org/10.1056/NEJMoa061759)

Prasad RB, Ahlqvist E \& Groop L 2019 Heterogeneity of diabetes - an Indian perspective. Diabetes and Metabolic Syndrome 13 3065-3067. (https:// doi.org/10.1016/j.dsx.2018.07.001)

Prasad RB, Asplund O, Shukla SR, Wagh R, Kunte P, Bhat D, Parikh M, Shah M, Phatak S, Käräjämäki A, et al. 2022 Subgroups of young type 2 diabetes in india reveal insulin deficiency as a major driver.Diabetologia 65 65-78. (https://doi.org/10.1007/s00125-021-05543-y)

Rabkin R, Ryan MP \& Duckworth WC 1984 The renal metabolism of insulin. Diabetologia 27 351-357. (https://doi.org/10.1007/BF00304849)
Regnell SE \& Lernmark Å 2017 Early prediction of autoimmune (type 1) diabetes. Diabetologia 60 1370-1381. (https://doi.org/10.1007/s00125017-4308-1)

Saxena R, Gianniny L, Burtt NP, Lyssenko V, Giuducci C, Sjogren M, Florez JC, Almgren P, Isomaa B, Orho-Melander M, et al. 2006 Common single nucleotide polymorphisms in TCF7L2 are reproducibly associated with type 2 diabetes and reduce the insulin response to glucose in nondiabetic individuals. Diabetes 55 2890-2895. (https:// doi.org/10.2337/db06-0381)

Schernthaner G, Matthews DR, Charbonnel B, Hanefeld M, Brunetti P \& Quartet [corrected] Study Group 2004 Efficacy and safety of pioglitazone versus metformin in patients with type 2 diabetes mellitus: a double-blind, randomized trial. Journal of Clinical Endocrinology and Metabolism 89 6068-6076. (https://doi.org/10.1210/ jc.2003-030861)

Shelgikar KM, Hockaday TD \& Yajnik CS 1991 Central rather than generalized obesity is related to hyperglycaemia in Asian Indian subjects. Diabetic Medicine 8 712-717. (https://doi. org/10.1111/j.1464-5491.1991.tb01689.x)

Slieker RC, Donnelly LA, Fitipaldi H, Bouland GA, Giordano GN, Åkerlund M, Gerl MJ, Ahlqvist E, Ali A, Dragan I, et al. 2021 Replication and cross-validation of type 2 diabetes subtypes based on clinical variables: an IMI-RHAPSODY study. Diabetologia 64 1982-1989. (https://doi.org/10.1007/s00125-021-05490-8)

Spoto B, Pisano A \& Zoccali C 2016 Insulin resistance in chronic kidney disease: a systematic review. American Journal of Physiology: Renal Physiology 311 F1087-F1108. (https://doi.org/10.1152/ ajprenal.00340.2016)

Szendroedi J, Saxena A, Weber KS, Strassburger K, Herder C, Burkart V, Nowotny B, Icks A, Kuss O, Ziegler D, et al. 2016 Cohort profile: the German Diabetes Study (GDS). Cardiovascular Diabetology 15 59-59. (https://doi.org/10.1186/s12933-016-0374-9)

Tanabe H, Saito H, Kudo A, Machii N, Hirai H, Maimaituxun G, Tanaka K, Masuzaki H, Watanabe T, Asahi K, et al. 2020 Factors associated with risk of diabetic complications in novel cluster-based diabetes subgroups: a Japanese retrospective cohort study. Journal of Clinical Medicine 9 2083. (https://doi.org/10.3390/jcm9072083)

Tuomi T, Carlsson A, Li H, Isomaa B, Miettinen A, Nilsson A, Nissen M, Ehrnstrom BO, Forsen B, Snickars B, et al. 1999 Clinical and genetic characteristics of type 2 diabetes with and without GAD antibodies. Diabetes 48 150-157. (https://doi.org/10.2337/diabetes.48.1.150)

Tuomi T, Santoro N, Caprio S, Cai M, Weng J \& Groop L 2014 The many faces of diabetes: a disease with increasing heterogeneity. Lancet 383 1084-1094. (https://doi.org/10.1016/S0140-6736(13)62219-9)

Udler MS, Kim J, von Grotthuss M, Bonas-Guarch S, Cole JB, Chiou J, Christopher D. Anderson on behalf of METASTROKE and the ISGC, Boehnke M, Laakso M, et al. 2018 Type 2 diabetes genetic loci informed by multi-trait associations point to disease mechanisms and subtypes: a soft clustering analysis. PLoS Medicine 15 e1002654.(https://doi. org/10.1371/journal.pmed.1002654)

Unwin N, Shaw J, Zimmet P \& Alberti KG 2002 Impaired glucose tolerance and impaired fasting glycaemia: the current status on definition and intervention. Diabetic Medicine 19 708-723. (https://doi.org/10.1046/ j.1464-5491.2002.00835.x)

Wagner R, Heni M, Tabak AG, Machann J, Schick F, Randrianarisoa E, Hrabe de Angelis M, Birkenfeld AL, Stefan N, Peter A, et al. 2021 Pathophysiology-based subphenotyping of individuals at elevated risk for type 2 diabetes. Nature Medicine 27 49-57. (https://doi.org/10.1038/ s41591-020-1116-9)

Wallace TM, Levy JC \& Matthews DR 2004 Use and abuse of HOMA modeling. Diabetes Care 27 1487-1495. (https://doi.org/10.2337/ diacare.27.6.1487)

Welsh GI, Hale LJ, Eremina V, Jeansson M, Maezawa Y, Lennon R, Pons DA, Owen RJ, Satchell SC, Miles MJ, et al. 2010 Insulin signaling to the glomerular podocyte is critical for normal kidney function. Cell Metabolism 12 329-340. (https://doi.org/10.1016/j.cmet.2010.08.015) https://joe.bioscientifica.com

https://doi.org/10.1530/JOE-20-0596 (c) 2021 Society for Endocrinology Published by Bioscientifica Ltd. Printed in Great Britain 
Xiong XF, Yang Y, Wei L, Xiao Y, Li L \& Sun L 2021 Identification of two novel subgroups in patients with diabetes mellitus and their association with clinical outcomes: a two-step cluster analysis. Journal of Diabetes Investigation 12 1346-1358. (https://doi.org/10.1111/ jdi.13494)

Yajnik CS \& Yudkin JS 2004 The Y-Y paradox. Lancet 363 163. (https://doi. org/10.1016/S0140-6736(03)15269-5)

Zaharia OP, Strassburger K, Strom A, Bonhof GJ, Karusheva Y, Antoniou S, Bodis K, Markgraf DF, Burkart V, Mussig K, et al. 2019 Risk of diabetes-associated diseases in subgroups of patients with recent-onset diabetes: a 5-year follow-up study. Lancet: Diabetes and Endocrinology 7 684-694. (https://doi.org/10.1016/S22138587(19)30187-1)
Zaharia OP, Strassburger K, Knebel B, Kupriyanova Y, Karusheva Y, Wolkersdorfer M, Bódis K, Markgraf DF, Burkart V, Hwang JH, et al. 2020 Role of Patatin-like phospholipase domain-containing 3 gene for hepatic lipid content and insulin resistance in diabetes. Diabetes Care 43 2161-2168. (https://doi.org/10.2337/dc20-0329)

Zou X, Zhou X, Zhu Z \& Ji L 2019 Novel subgroups of patients with adultonset diabetes in Chinese and US populations. Lancet: Diabetes and Endocrinology 7 9-11. (https://doi.org/10.1016/S2213-8587(18)30316-4)

Zou H, Duan W, Zhang Z, Chen X, Lu P \& Yu X 2020 The circulating ANGPTL8 levels show differences among novel subgroups of adult patients with diabetes and are associated with mortality in the subsequent 5 years. Scientific Reports 10 12859. (https://doi.org/10.1038/ s41598-020-69091-y)

Received in final form 20 September 2021

Accepted 9 November 2021

Accepted Manuscript published online 16 November 2021 (c) 2021 Society for Endocrinology Published by Bioscientifica Ltd. 